\title{
L'OCT plein champ
}

\section{Arnaud Dubois, Claude Boccara}

> La tomographie par cohérence optique, plus communément appelée OCT (optical coherence tomography), est une technique d'imagerie non invasive des milieux biologiques à l'échelle du micromètre dont l'impact le plus remarquable concerne l'ophtalmologie. L'OCT «plein champ » est une approche originale de l'OCT, proposée et améliorée au fil des ans par notre équipe. Après avoir exposé le principe de l'OCT plein champ, nous détaillerons ses performances en soulignant les avantages et les inconvénients par rapport à I'OCT classique. Les potentialités de cette technique seront illustrées par quelques exemples d'applications dans les domaines de l'embryologie, la biologie du développement et l'ophtalmologie. Enfin, nous présenterons les développements en cours pour l'imagerie à très haute résolution in vivo, pour accroître la profondeur d'imagerie dans les milieux fortement diffusants, ou encore exploiter de nouvelles sources de contraste comme la biréfringence optique. <

\section{L'OCT et ses variantes}

L'OCT est fondée sur l'interférométrie en lumière polychromatique dite faiblement cohérente. Dans cette introduction, nous citons différentes variantes de I'OCT, ce qui permettra au lecteur de suivre la bibliographie sur ce thème. Classiquement, l'OCT produit des images en coupe axiale (mode B comme en imagerie échographique), la dimension transverse de l'image étant obtenue par balayage d'un faisceau lumineux et la dimension axiale (la profondeur), en faisant varier la différence de marche dans l'interféromètre [1-3]. Depuis son invention au début des années 1990, l'OCT a connu de nombreux développements. Dans l'OCT spectrale (ou OCT de Fourier), le balayage de la différence de marche est remplacé par une mesure en parallèle du spectre du signal interférométrique, ce qui conduit

Article reçu le 2 mars 2006, accepté le 14 juin 2006.

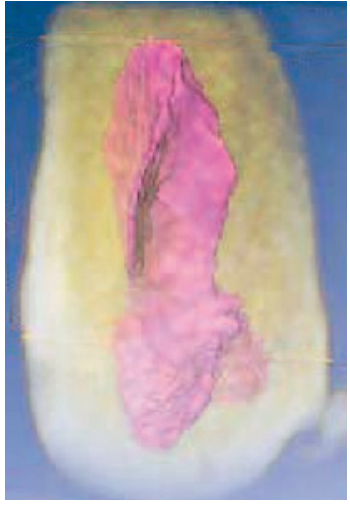

Laboratoire d'Optique Physique, École Supérieure de Physique et Chimie Industrielles de la Ville de Paris, CNRS UPR A0005, 10 , rue Vauquelin, 75005 Paris, France.

boccara@optique.espci.fr

à une réduction spectaculaire du temps d'acquisition [4-6]. L'OCT en lumière polarisée, capable de révéler les propriétés optiques de biréfringence, apporte des informations supplémentaires sur la structure et la composition des tissus [7-9]. L'OCT Doppler, permettant de mesurer des vitesses de déplacement, a ouvert la voie à l'étude des écoulements sanguins de très petite vitesse $[10,11]$. L'OCT plein champ est une approche conceptuellement différente de l'OCT classique qui utilise un capteur d'image tel qu'une caméra CCD. Des coupes transverses sont obtenues sans balayage à partir d'une combinaison d'images acquises au moyen d'un microscope interférométrique éclairé par une source de lumière thermique $[12,13]$.

\section{Principe et performances de l'OCT plein champ}

Le dispositif expérimental de l'OCT plein champ (Figure 1) est fondé sur un interféromètre de Michelson avec des objectifs de microscope placés dans ses deux bras, configuration dite de Linnik. Le système d'illumination utilise une lampe halogène à filament de tungstène. À cause du spectre très large de cette lumière, des interférences se produisent à condition que la différence de marche dans l'interféromètre soit très proche de zéro (Encadré). La longueur du bras de référence de l'interféromètre détermine, au niveau de l'objet à imager, une tranche d'épaisseur $\Delta z$ fixée par la largeur du spectre de la source lumineuse. Seule la lumière réfléchie par les structures de l'objet situées dans cette tranche crée des interférences. Ces structures peuvent être imagées si l'on parvient à extraire l'amplitude du signal d'interférence. Pour cela, on combine plusieurs images interférométriques, acquises au moyen d'un 
détecteur matriciel CCD, et déphasées entre elles grâce à l'oscillation du miroir de référence [13]. On obtient ainsi, par calculs, une image du volume de cohérence en temps réel (cadence de quelques Hertz), l'image d'une fine tranche orientée transversalement.

La résolution transverse des images d'OCT plein champ est similaire à celle des images fournies par un microscope, soit environ $1 \mu \mathrm{m}$. En OCT traditionnelle, la résolution transverse est largement inférieure car une grande profondeur de champ est requise pour produire des images orientées axialement sans réajuster la mise au point. Notons que des images transverses peuvent également être obtenues en OCT traditionnelle (mais pas en OCT spectrale) en balayant un faisceau cohérent dans le plan transverse [1416]. La résolution transverse peut alors être élevée.

En OCT, la résolution axiale est indépendante de la résolution transverse : elle est déterminée par la largeur du spectre de la source lumière. Dans notre cas, le spectre effectif est limité par la réponse spectrale de la caméra CCD en silicium (DALSA 1M15). Ce spectre est centré à la longueuer d'onde $\lambda=0,8 \mu \mathrm{m}$ et présente une largeur à mi-hauteur $\Delta \lambda=300 \mathrm{~nm}$. La résolution axiale, donnée par la formule [3] :

$$
\Delta z=\frac{2 \ln 2}{n \pi}\left(\frac{\lambda^{2}}{\Delta \lambda}\right),
$$

vaut alors $\Delta z=0,7 \mu \mathrm{m}$ lorsque l'objet est constitué d'eau essentiellement (indice de réfraction $n=1,33$ ). L'utilisation d'objectifs à immersion à eau permet de maintenir la résolution axiale quelle que soit la profondeur explorée [17]. Avec une simple lampe halogène, on atteint une résolution identique à celle obtenue en OCT à balayage avec un laser émettant des impulsions de 5 femtosecondes [18]. Le spectre d'une source thermique est beaucoup plus lisse, ce qui évite les artefacts dans les images résultants de la présence de lobes latéraux dans l'interférogramme.

Finalement, I'OCT plein champ offre une résolution spatiale isotrope de $1 \mu \mathrm{m}$ sans avoir recours, comme en microscopie confocale, à des objectifs de microscope de très grande ouverture numérique qui limitent l'imagerie en profondeur à cause des aberrations optiques.

La sensibilité de détection et la dynamique de l'OCT sont capitales pour réaliser des images dans la profondeur des milieux biologiques malgré la diffusion importante de la lumière. Les performances de notre instrument dépendent essentiellement de la capacité de charge des pixels du capteur CCD, c'est-à-dire le nombre de photoélectrons qui peut être stocké dans le «puit» de chaque pixel. La faiblesse de cette capacité de charge $\left(10^{5}\right.$ à $\left.10^{6}\right)$ peut être compensée par l'accumulation de plusieurs images et par le regroupement de pixels (binning). Nous parvenons ainsi à atteindre une sensibilité remarquable de l'ordre de $90 \mathrm{~dB}$ : le nombre de $\mathrm{dB}$ est égal à 10 fois le logarithme du rapport de une intensité incidente sur l'intensité réfléchie; par exemple, un coefficient de réflexion de $10^{-9}$ soit $90 \mathrm{~dB}$ est équivalent à un miroir qui, recevant un milliard de photons, n'en réfléchirait qu'un seul.

Le détecteur d'image CCD utilisé en OCT plein champ permet l'acquisition du signal en parallèle par des millions de pixels à la cadence vidéo; en OCT plein champ, le temps d'acquisition de chaque pixel, égal au temps d'acquisition de l'image complète, est considérablement plus long que dans la technique à balayage. En OCT à balayage, le temps d'acquisition par pixel très bref évite le brouillage du signal d'interférence. Les mouvements de l'objet peuvent seulement engendrer des distorsions dans l'image qui peuvent être corrigées numériquement après l'acquisition [19]. Malgré ces inconvénients, I’OCT plein champ 
présente un avantage certain: sa très haute résolution spatiale obtenue avec une simple lampe halogène. Largement supérieure à celle des systèmes d'OCT utilisant des diodes superluminescentes, elle est comparable à celle obtenue avec des lasers femtosecondes ultra-brefs, sources lumineuses encore extrêmement sophistiquées et onéreuses [18]. En outre, l'OCT plein champ évite le recours à des systèmes de balayage de faisceau et de la différence de marche. L'OCT plein champ constitue une alternative particulièrement avantageuse à l'OCT à balayage pour les applications d'imagerie tridimensionnelle à très haute résolution spatiale de la structure interne d'objets biologiques quasi-statiques.

\section{Applications de l'OCT plein champ}

Les examens histologiques sont très courants en biologie et en médecine. Ils nécessitent une préparation parfois longue et délicate de l'échantillon avant l'observation au microscope. Une coloration est généralement nécessaire pour apporter du contraste à l'image. L'OCT plein champ peut être utilisée pour effectuer des examens histologiques beaucoup

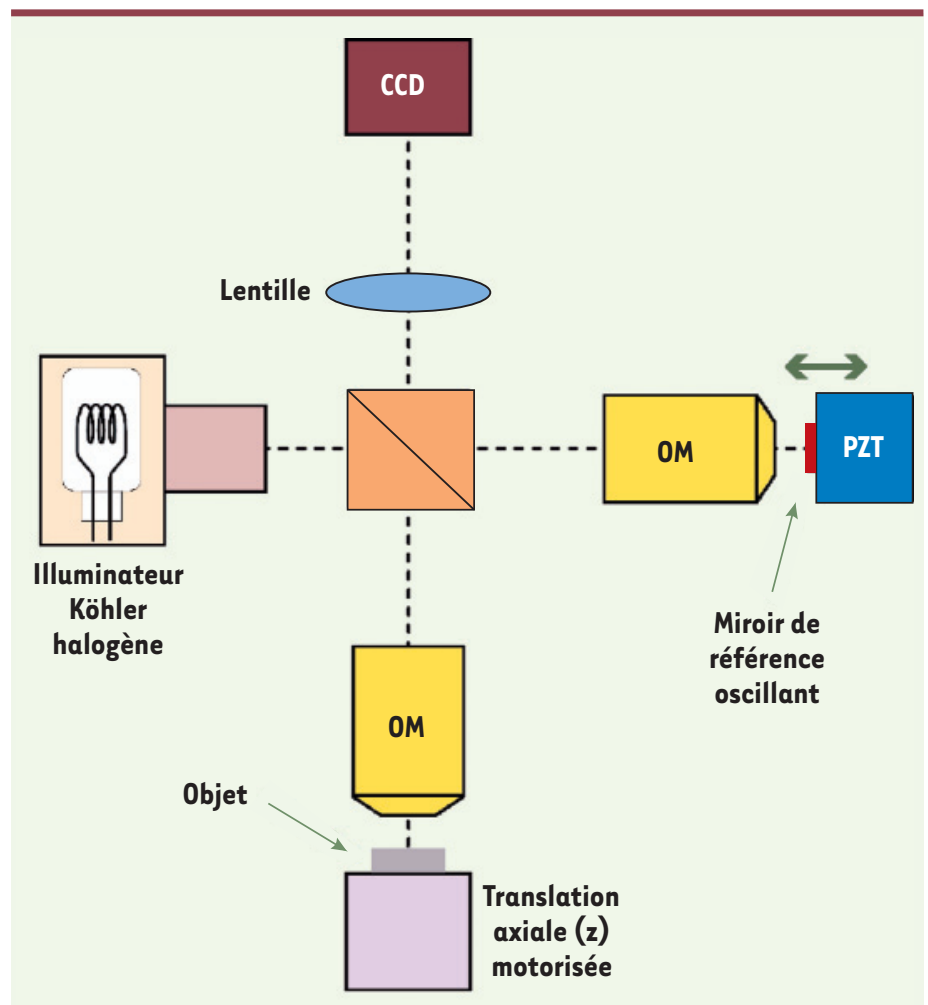

Figure 1. Schéma de principe de l'OCT plein champ. II s'agit d'un interféromètre de Michelson avec des objectifs de microscope (OM) à immersion à eau, éclairé au moyen d'une lampe halogène. Le miroir de référence constitue l'un des bras de l'interféromètre, la tranche de l'objet située à une profondeur susceptible d'interférer constitue le second bras. Les images tomographiques (coupes transverses) sont calculées à partir d'images interférométriques, enregistrées par une caméra CCD, et déphasées entre elles grâce à l'oscillation du miroir de référence induite par un système piézoélectrique (PZT). plus rapidement, sans préparation de l'échantillon et sans utiliser d'agent de contraste. À partir d'une pile d'images en coupe, on peut ensuite calculer des coupes selon des orientations quelconques. Des images en trois dimensions peuvent également être produites avec une résolution spatiale proche de celle de l'histologie standard. Nous avons utilisé l'OCT plein champ pour des études dans le domaine de l'embryologie. On peut voir sur la Figure 2 un exemple d'images réalisées chez le têtard de grenouille africaine Xenopus Laevis. L'animal (ex vivo) était placé dans une solution de DPBS (Dulbecco's phosphate buffered saline) pour conservation, et une pile de 500 images tomographiques a été enregistrée avec un pas de $0,5 \mu \mathrm{m}$. Diverses structures, notamment les noyaux et membranes de cellules, sont révélées. Cet animal modèle des batraciens pour la biologie du développement a été très utilisé pour démontrer les performances de I'OCT à haute résolution. Notre instrument a donné des images de qualité comparable (voire supérieure) à celles des systèmes d'OCT à balayage les plus performants [18].

L'embryon de souris est un modèle de choix pour l'étude du développement des mammifères. La Figure 3 montre des images d'un embryon âgé de 6,5 jours. L'OCT plein champ s'est avéré être un outil précieux pour l'étude des mécanismes régissant l'établissement de l'axe antéro-posterieur [20]. Outre un gain en temps considérable, I'OCT plein champ a permis d'éviter tout risque d'endommager ou de déformer l'embryon, avantage considérable par rapport aux techniques classiques nécessitant un découpage en fines tranches après fixation et imprégnation dans une résine.

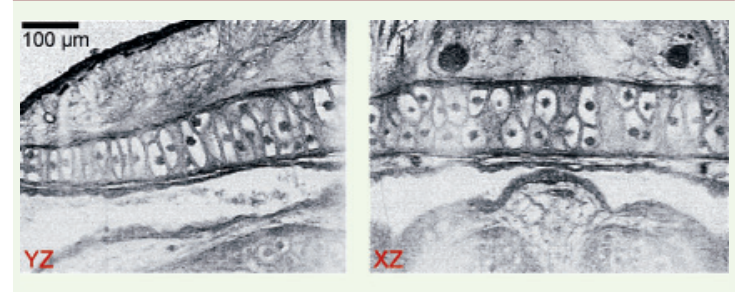

Figure 2. Images tomographiques axiales réalisées dans la tête du têtard de grenouille africaine Xenopus Laevis (in vitro). L'axe Z est l'axe optique de l'objectif de microscope. Les images sont prises sous forme de piles de coupes «en face » dites XY. A partir de ces piles, nous reconstituons des coupes, ici dans les plans $Y Z$ et $Y Z$ à l'aide du programme Image J mis dans le domaine publique par le $\mathrm{NIH}$. La résolution isotrope de 1 micromètre de l'OCT plein champ permet la visualisation de structures subcellulaires. Différents stades de la mitose peuvent être observés. Des tissus fortement contrastés apparaissent tels que l'épiderme, les nerfs olfactifs et les mélanocytes. 
En collaboration avec des opthalmologistes, nous avons réalisé des images par OCT plein champ des différents éléments constitutifs des segments antérieurs et postérieurs de l'œil de diverses espèces animales (rat, lapin, chat et porc) [21, 22]. Un exemple d'images de la cornée, du cristallin et de la rétine de l'œil de rat est présenté sur la Figure 4. Les échantillons prélevés étaient placés dans un récipient rempli d'une solution de DPBS. Dans la cornée, on peut discerner les cellules épithéliales. Les membranes de Bowman et de Descemet sont visibles, ainsi que la structure du stroma contenant des kératocytes. Notons que le contour irrégulier de la membrane basale résulte vraisemblablement d'un endommagement de l'échantillon lors du transport. Dans le cristallin, la capsule, la couche de cellules épithéliales et les fibres corticales sont révélées. Enfin, les différentes couches de la rétine sont parfaitement identifiables. Le réseau de fibres nerveuses peut être visualisé sur l'image transverse (XY), ainsi que des cellules ganglionnaires sur la coupe axiale.

\section{Développements en cours}

Les propriétés de réflexion et d'absorption de la lumière par les tissus sont la source de contraste des images d'OCT, révélatrices d'informations morphologiques. Nous développons actuellement une nouvelle technique d'OCT plein champ capable de mesurer également la biréfringence, une propriété optique qui se traduit par une modification de la polarisation de la lumière. La plupart des tissus biologiques présentent de la biréfringence liée à la présence de composants comme le collagène, la kératine, ou encore le glucose. Ce mode de contraste apportera des informations supplémentaires sur la structure et la composition tissulaire.

La profondeur pouvant être explorée par l'OCT est déterminée par les phénomènes de diffusion et d'absorption de la lumière [23]. La diffusion diminue rapidement quand la longueur d'onde augmente depuis le visible jusqu'à l'infrarouge. L'absorption dans les milieux biologiques est dominée par diverses bandes de résonance. Dans le proche infrarouge, autour de $0,8 \mu \mathrm{m}$, l'absorption est relativement faible. La diffusion est alors le mécanisme d'atténuation largement prédominant. À des longueurs d'ondes plus grandes, au-delà de $2 \mu \mathrm{m}$, la diffusion est minimale mais l'absorption par l'eau devient prédominante. Des études comparatives, dans les milieux fortement diffusants, ont montré une meilleure pénétration et un meilleur contraste des images autour de la longueur d'onde de $1,3 \mu \mathrm{m}$ plutôt que 0,8 $\mu \mathrm{m}$ [24]. C'est pourquoi nous développons actuellement un nouveau système d'OCT plein champ basé sur une caméra infrarouge utilisant un capteur en arséniure de gallium indium (InGaAs) dont la réponse spectrale se situe entre $0,9 \mu \mathrm{m}$ et $1,7 \mu \mathrm{m}^{1}$.

L'un de nos autres projets majeurs est de réaliser un système d'OCT plein champ très rapide, afin de pouvoir étudier des objets susceptibles de bouger rapidement. Une première solution consiste à utiliser une caméra à grande vitesse d'acquisition. Pour atteindre

${ }^{1}$ Dubois A, Moneron G, Boccara AC. Thermal-light full-field optical coherence tomography in the $1.2 \mu \mathrm{m}$ wavelength region (soumis pour publication). une dynamique suffisante, l'accumulation temporelle d'images est alors remplacée par une sommation spatiale en regroupant des pixels (binning). Le temps d'acquisition d'une image tomographique peut ainsi être descendu à environ 10 millisecondes (au lieu de 1 seconde) [25]. Un fonctionnement encore plus rapide peut être atteint en utilisant un éclairage très bref qui fige le mouvement de l'objet. Cet éclairage est obtenu au moyen d'une lampe flash à arc au xénon. On utilise deux caméras CCD pour enregistrer simultanément deux images interférométriques en opposition de phase dont la différence donne une image tomographique. Pour augmenter la faible dynamique des capteurs CCD, les images sont enregistrées avec des caméras comportant un très grand nombre de pixels qui sont ensuite regroupés par paquets (binning). Le principe de cette technique a été validé récemment [26].

Le développement d'un système d'OCT plein champ très rapide est motivé par un grand nombre d'appli-

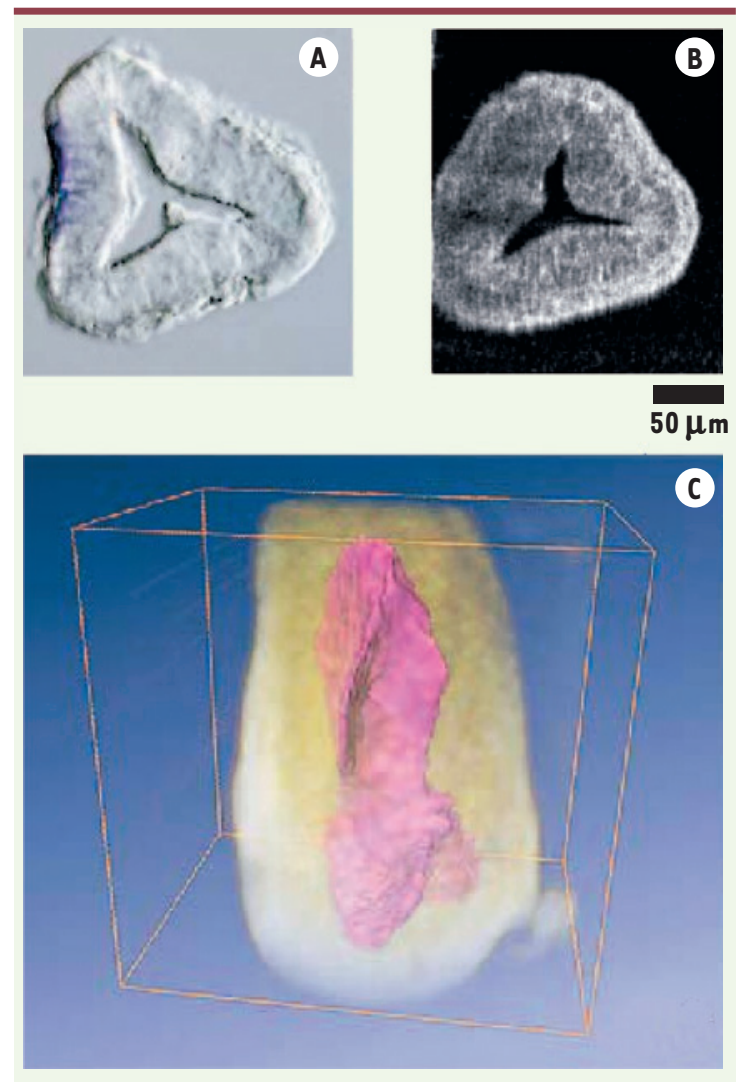

Figure 3. Images d'un embryon de souris agé de 6,5 jours. A. Coupe histologique observée au microscope de Nomarski. B. Image tomographique obtenue par OCT plein champ à partir d'un embryon entier sans préparation. C. Image 3D de la cavité proamniotique reconstruite à partir d'une pile d'images tomographiques (comme l'image $\boldsymbol{B}$ ). 


\section{GLOSSAIRE}

Binning : ajout de pixels voisins pour augmenter les signaux au detriment de la résolution.

Biréfringence : différence d'indice de réfraction pour deux polarisations orthogonales de la lumière.

Bras de référence, bras objet: les deux trajets de l'onde après division.

CCD (charge coupled device): matrice de détecteurs solides (silicium en général) qui remplace, par exemple, les fims photographiques dans les appareils photographiques numériques.

Différence de marche : chemin parcouru par la lumière (en fait temps/vitesse).

Diodes superluminescentes: ce sont des structures semiconductrices qui émettent de la lumère polycromatiques (à la différence d'un laser) à partir d'un canal de taille très étroite (comme un laser à semiconducteur).
Interférométrie : utilisation d'un interféromètre (Encadré) capable de produire des interférences par division d'une onde incidente puis recombinaison (ex : l'interferomètre de Michelson). Isotrope: un phénomène est isotrope s'il est identique dans toutes les directions de l'espace.

Luminance d'une source de lumière : puissance émise par unité de surface et d'angle solide.

Résolution axiale et transverse : la résolution d'un microscope est la distance minimum de séparation deux objets de très petite taille respectivement le long de l'axe ou dans le plan perpendiculaire à l'axe.

Source thermique : souce de lumière obtenue par chauffage d'un filament (exemple : lampe halogène).
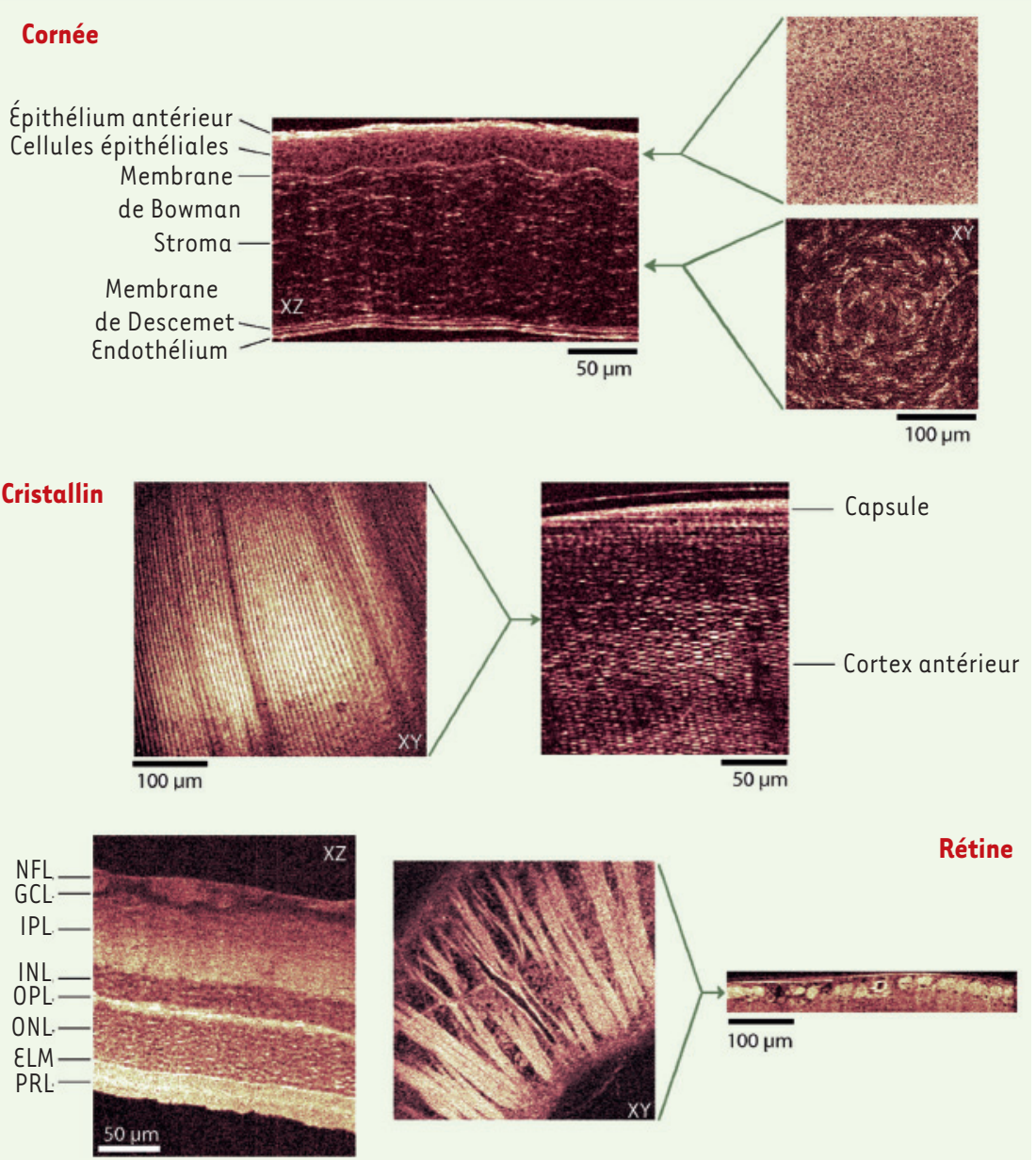

Rétine

Figure 4. Images obtenue par OCT plein champ de cornée, cristallin et rétine prélevés dans un œil de rat. NFL : couche des fibres nerveuses; GCL : couche des cellules ganglionnaires; IPL : couche plexiforme interne; INL : couche granulaire interne ; OPL : couche plexiforme externe; ONL : couche granulaire externe; $\varepsilon L M$ : membrane limitante externe; PRL : couche des photorécepteurs. cations biologiques in vivo. L'imagerie de l'œil humain est une des applications visées prioritaire. Dans notre futur instrument d'OCT ultrarapide sera inséré un système comportant un miroir déformable pour corriger les défauts optiques de l'œil en temps réel. La combinaison de l'OCT plein champ et de la technique d'optique adaptative issue de l'astronomie permettra l'observation de la rétine in situ à l'échelle cellulaire.

\section{SUMMARY}

\section{Full-field OCT}

Optical coherence tomography (OCT) is an emerging technique for imaging of biological media with micrometerscale resolution, whose most significant impact concerns ophthalmology. Since its introduction in the early 1990's, OCT has known a lot of improvements and sophistications. Full-field OCT is our original approach of OCT, based on white-light interference microscopy. Tomographic images are obtained by combination of interferometric images recorded in parallel by a detector array such as a CCD camera. Whereas conventional OCT produces B-mode (axially-oriented) images like ultrasound imaging, full-field OCT acquires tomographic images in the en face (transverse) orientation. Full-field OCT is an alternative method to con- 
ventional OCT to provide ultrahigh resolution images $(\sim 1 \mu \mathrm{m})$, using a simple halogen lamp instead of a complex laser-based source. Various studies have been carried, demonstrating the performances of this technology for three-dimensional imaging of ex vivo specimens. Fullfield OCT can be used for non-invasive histological studies without sample preparation. In vivo imaging is still difficult because of the object motions. A lot of efforts are currently devoted to overcome this limitation. Ultra-fast full-field OCT was recently demonstrated with unprecedented image acquisition speed, but the detection sensitivity has still to be improved. Other research directions include the increase of the imaging penetration depth in highly scattering biological tissues such as skin, and the exploitation of new contrasts such as optical birefringence to provide additional information on the tissue morphology and composition. $\diamond$

\section{RéFÉRENCES}

1. Huang D, Swanson EA, Lin CP, et al. Optical coherence tomography. Science 1991 ; $254: 1178-81$.

2. Fujimoto JG, Brezinski ME, Tearney GJ, et al. Optical biopsy and imaging using optical coherence tomography. Nat Med $1995 ; 1: 970-2$.

3. Fercher AF. Optical coherence tomography. J Biomed Opt 1996; 1: 157-73.

4. Wojtkowski M, Leitgeb R, Kowalczyk A, et al. In vivo human retinal imaging by Fourier domain optical coherence tomography. J Biomed Opt $2002 ; 7: 457-63$.

5. Nassif $\mathrm{N}$, Cense $\mathrm{B}$, Park BH, et al. In vivo human retinal imaging by ultrahigh-speed spectral domain optical coherence tomography. Opt Lett $2004 ; 29: 480-2$.

6. Leitgeb RA, Drexler W, Unterhuber A, et al. Ultrahigh resolution Fourier domain optical coherence tomography. Opt Express $2004 ; 12: 2156-65$.

7. De Boer JF, Milner TE, Van Gemert MJC, Nelson JS. Two-dimensional birefringence imaging in biological tissue by polarization sensitive optical coherence tomography. Opt Lett 1997 ; $22: 934-6$.

8. Park BH, Saxer C, Srinivas SM, et al. In vivo burn depth determination by highspeed fiberbased polarization sensitive optical coherence tomography. J Biomed Opt $2001 ; 6$ : 474-9.

9. Jiao S, Yao G, Wang LV. Two-dimensional depth-resolved Mueller matrix of biological tissue measured with double-beam polarization-sensitive optical coherence tomography. Opt Lett $2002 ; 27: 101-3$.

10. Chen $Z$, Miller TE, Srinivas $S$, et al. Noninvasive imaging of in vivo blood flow velocity using optical Doppler tomography. Opt Lett $1997 ; 22$ : 1119-21.

11. Westphal V, Yazdanfar S, Rollins AM, Izatt JA. Real-time, high velocity-resolution color Doppler optical coherence tomography. Opt Lett $2002 ; 27: 34-6$.
12. Vabre L, Dubois A, Boccara AC. Thermal-light full-field optical coherence tomography. Opt Lett $2002 ; 27: 530-2$.

13. Dubois A, Grieve K, Moneron G, et al. Ultrahigh-resolution full-field optical coherence tomography. Appl Opt $2004 ; 43: 2874-83$.

14. Izatt JA, Hee MR, Owen GM, et al. Optical coherence microscopy in scattering media. Opt Lett $1994 ; 19: 590-2$.

15. Podoleanu AG, Rogers JA, Jackson DA, Dunne S. Three dimensional OCT images from retina and skin. Opt Express $2000 ; 7: 292-8$.

16. Hitzenberger CK, Trost P, Lo PW, Zhou Q. Three-dimensional imaging of the human retina by high-speed optical coherence tomography. Opt Express $2003 ; 11: 2753-61$.

17. Hitzenberger CK, Baumgartner A, Drexler W, Fercher AF. Dispersion effects in partial coherence interferometry: implications for intraocular ranging. J Biomed Opt 1999; $4: 144-51$.

18. Drexler W, Morgner U, Kärtner, et al. In vivo ultrahigh-resolution optical coherence tomography. Opt Lett $1999 ; 24$ : 1221-3.

19. Swanson EA, Izatt JA, Hee MR, et al. In vivo retinal imaging by optical coherence tomography. Opt Lett 1993 ; 18 : 1864-6.

20. Perea-Gomez A, Moreau A, Camus A, et al. Initiation of gastrulation in the mouse embryo is preceded by an apparent shift in the orientation of the anterior-posterior axis. Curr Biol 2004 ; 14 : 197-207.

21. Grieve K, Paques M, Dubois A, et al. Ocular tissue imaging using ultrahigh-resolution full-field optical coherence tomography. Invest Ophthalmol Vis Sci $2004 ; 45: 4126-31$.

22. Dubois A, Moneron G, Grieve K, Boccara AC. Three-dimensional cellularlevel imaging using full-field optical coherence tomography. Phys Med Biol $2004 ; 49$ : 1227-34.

23. Schmitt J, Xiang SH, Yung KM. Differential absorption imaging with optical coherence tomography. J Opt Soc Am A 1998; 15 : 2288-96.

24. Schmitt JM, Knuttel A, Yadlowsky M, Eckhaus MA. Optical-coherence tomography of a dense tissue : statistics of attenuation and backscattering. Phys Med Biol 1994 ; 39 : 1705-20.

25. Grieve $K$, Dubois $A$, Simonutti $M$, et al. In vivo anterior segment imaging in the rat eye with high speed white light full-field optical coherence tomography. Opt Express $2005 ; 13: 6286-95$.

26. Moneron G, Boccara AC, Dubois A. Stroboscopic ultrahigh-resolution fullfield optical coherence tomography. Opt Lett $2005 ; 30$ : 1351-3.

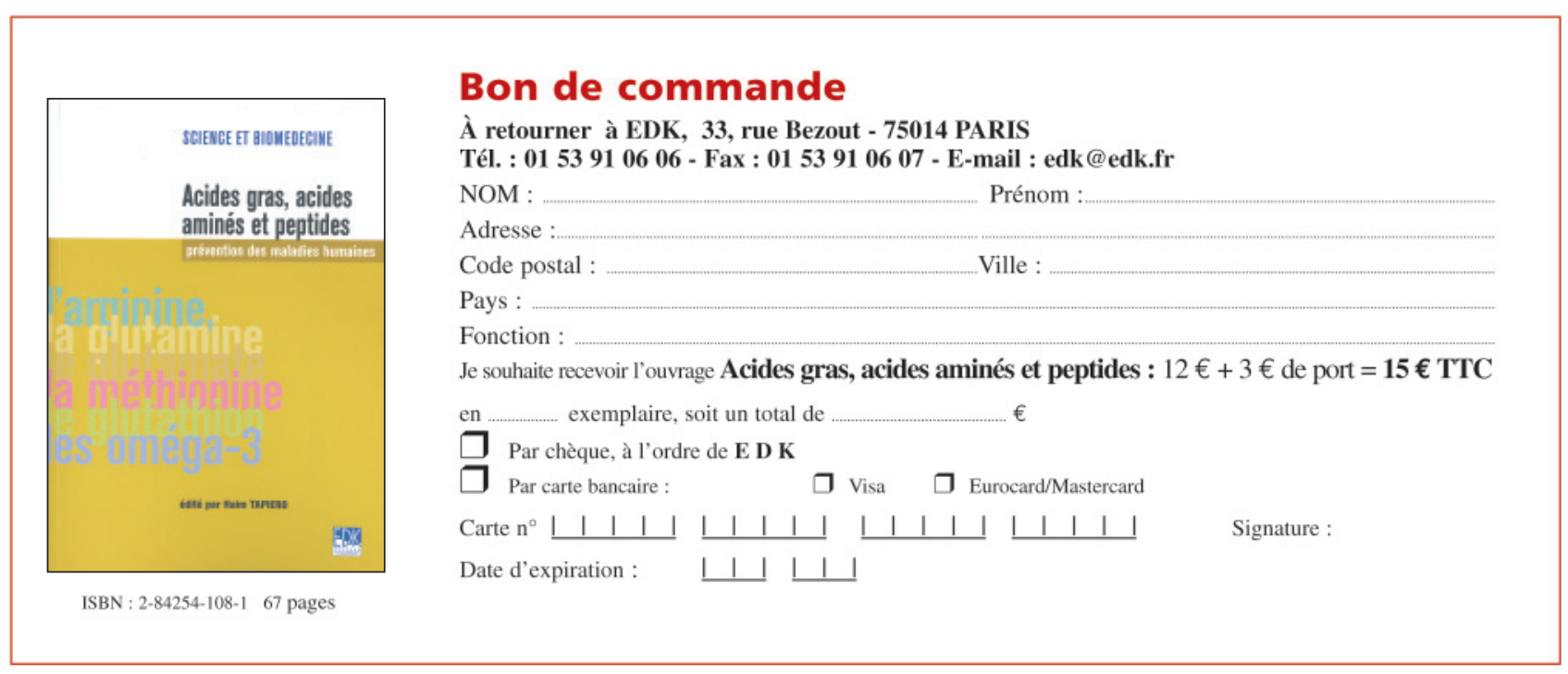

